\title{
Pratiques
}

Linguistique, littérature, didactique

$159-160 \mid 2013$

Le figement en débat

\section{Extensions lexicales et figures vives : une frontière essentielle}

Michele Prandi

\section{(2) OpenEdition}

Journals

Édition électronique

URL : http://journals.openedition.org/pratiques/2826

DOI : 10.4000 /pratiques. 2826

ISSN : 2425-2042

Éditeur

Centre de recherche sur les médiations (CREM)

Édition imprimée

Date de publication : 15 décembre 2013

Pagination : 55-68

Référence électronique

Michele Prandi, «Extensions lexicales et figures vives : une frontière essentielle », Pratiques [En ligne], 159-160 | 2013, mis en ligne le 30 juin 2016, consulté le 01 mai 2019. URL : http://

journals.openedition.org/pratiques/2826 ; DOI : 10.4000/pratiques.2826

(c) Tous droits réservés 


\title{
Extensions lexicales et figures vives: une frontière essentielle
}

\author{
Michele Prandi
}

Université de Gênes

Depuis Quintilien, la tradition rhétorique distingue deux couples de familles de tropes : la métaphore et la «nebula métonymique » (Bonhomme 2006) d'une part, les figures vives et les extensions lexicales d'autre part. Tout en possédant des propriétés distinctives caractéristiques, les différentes familles sont censées former une unité de fond. En premier lieu, la métaphore et la métonymie sont considérées comme autant de formes de transfert qui ne se différencient que d'après le contenu du vecteur, ce qui présuppose que tous les tropes sont des formes de transfert. Ensuite, les extensions lexicales sont censées être l'aboutissement d'un processus de cristallisation de figures autrefois vives, ce qui présuppose que les extensions et les figures partagent la même structure conceptuelle essentielle : en particulier, le trope se résout en tout cas dans un affaiblissement du contenu du foyer, sur lequel se concentre la pression conceptuelle dégagée par le trope. Comme nous le verrons, les approches les plus récentes n'ont pas changé en profondeur ce tableau. En particulier, elles ont gardé les deux idées de fond - la différentiation dans la continuité de l'univers tropique - et les présupposés qui les fondent : 1'association entre trope et transfert d'une part, l'idée que la pression conceptuelle n'investit que le foyer d'autre part.

Dans cet essai, je me propose de remettre en question les présupposés sur l'unité structurale des tropes qui, comme nous le verrons ( $\$ 2.1)$, sont l'héritage d'une conception essentiellement substitutive des figures. Si nous allons au-delà d'une conception substitutive, la relation postulée par la tradition entre métaphore et métonymie, extension lexicale et figure est bouleversée, ce qui nous amènera à redessiner la topographie du champ tropologique. En premier lieu, le transfert de concepts n'est pas la propriété commune de tous les tropes mais la propriété distinctive et définitoire de la métaphore. Ensuite, la pression conceptuelle dégagée par le trope ne frappe pas nécessairement le foyer : en présence de métonymie, c'est toujours le foyer qui est mis sous pression; en présence de métaphore, par contre, si l'extension et la substitution agissent sur le foyer, la projection sollicite la teneur. Les conséquences sur la description des tropes sont profondes. La métaphore et la métonymie se présentent comme des structures conceptuelles et lin- 
guistiques profondément différentes, dont les retombées sur la conceptualisation sont incommensurables. Également, la métaphore vive et l'extension lexicale apparaissent comme deux structures spécifiques ayant des racines autonomes et répondant à des fonctions irréductibles, ce qui enlève beaucoup de force à l'idée d'une « carrière de la métaphore » (Bowdle \& Gentner 2005) de la figure vive à l'extension lexicale.

\section{Le champ tropologique}

Les expressions au contenu conflictuel offrent un observatoire privilégié tant sur la structure des tropes que sur la différence entre métaphore et métonymie. En plus, elles permettent de mettre au feu la question de la pression conceptuelle dégagée par le trope, qui frappe tantôt le foyer étranger et tantôt la teneur cohérente.

\subsection{La structure conceptuelle du trope}

Une expression conflictuelle présente deux constituants conceptuels : un cadre cohérent avec le texte ou la situation discursive, et un foyer incohérent (Black 1954). L'énoncé La lune rêve (Baudelaire), par exemple, décrit un paysage ; la lune est un constituant cohérent de ce paysage, alors que le verbe rêver y transfère un concept étranger.

La structure conceptuelle d'une figure, cependant, ne se réduit pas aux constituants ouverts du conflit, car elle s'ouvre à des constituants couverts ${ }^{(1)}$. Dans l'expression Passe un manteau de velours (Butor), par exemple, l'expression un manteau se réfère par métonymie à une personne qui n'est pas nommée dans l'expression. Les constituants conceptuels de cette métonymie ne sont donc pas le foyer et le cadre, mais le foyer et un double couvert - la personne - qui forme avec lui une relation paradigmatique in absentia. Dans l'énoncé La lune rêve (Baudelaire), tant le foyer rêver que le cadre la lune entrent chacun dans une opposition paradigmatique avec un double couvert : le verbe avec un état cohérent de la lune — par exemple, un voile de brouillard enveloppe la lune - et la lune avec l'être humain, sujet cohérent de rêver. Pour identifier de façon univoque les concepts engagés dans la figure il est donc nécessaire d'introduire deux termes spécifiques, excentriques par rapport au couple cadre - foyer : la teneur (Richards 1936) et le sujet de discours subsidiaire (Black 1954). Dans l'expression Passe un manteau de velours, le foyer manteau coïncide avec le sujet subsidiaire, alors que son double couvert - la personne fournit la teneur. Dans 1'expression La lune rêve, qui contient virtuellement deux métaphores, le foyer - rêver — introduit un premier sujet subsidiaire destiné à interagir avec une teneur couverte - par exemple, le brouillard qui contourne la lune - alors que le cadre - la lune — introduit une seconde teneur destinée à interagir avec un sujet subsidiaire couvert cohérent avec le foyer verbal : l'être humain.

\subsection{Métaphore et métonymie}

La distinction entre métaphore et métonymie à fait l'objet, dans les dernières décennies, d'un travail de mise au point remarquable. Commencé en France et axé surtout sur les figures vives (Henry 1971 ; Le Guern 1973 ; Prandi 1992 ; 2012 ; Bonhomme 1997 ; 2006), ce travail a été déplacé par les linguistes cognitivistes

(1) L'opposition entre ouvert et couvert traduit l'opposition entre overt et covert en anglais (voir par exemple Lounsbury 1956) 
d'expression anglaise (Lakoff 1987 ; Dirven \& Pörings 2002 ; Barcelona éd. 2003 ; Benczes et al. éds. 2011) dans le domaine des figures intégrées dans la pensée commune cohérente ( $\$ 2.2)$. Un point de continuité entre la réflexion contemporaine et les approches traditionnelles est le présupposé selon lequel tant la métaphore que la métonymie mettent en œuvre un transfert de concepts. Dans la tradition française, par exemple, Bonhomme $(1987 ; 2006: 59)$ parle explicitement de «transferts métonymiques »; dans 1'approche cognitive, Barcelona (2003: 4) définit la métonymie comme « a conceptual projection », où la projection est un processus qui présuppose le transfert. Le transfert d'un mot - le trope - fournit le genus proximum, alors que la differentia specifica est identifiée par le vecteur qui relie les concepts hétérogènes engagés : la contiguïté pour la métonymie, l'analogie pour la métaphore. L'expression Le travail des abeilles (Yourcenar) se réfère à la cire, qui est le résultat de leur travail : c'est un exemple de métonymie. Dans l'expression Le pont de nos bras (Apollinaire), le pli des bras est censé rappeler la courbure d'un pont.

Si nous focalisons l'attention sur les signifiés conflictuels, dans le style d'analyse que j'ai appelé grammaire philosophique (Prandi 1992 ; 2004), nous remarquons tout de suite une différence de fond entre métonymie et métaphore qui bouleverse l'idée traditionnelle. Alors que la métaphore valorise le conflit en transférant un concept dans un domaine étranger et en l'appliquant à un concept à son tour étranger, la métonymie dissout le conflit grâce à l'activation d'une relation cohérente entre les concepts en conflit; grâce à la relation cohérente qui franchit le fossé qui les sépare, chaque concept demeure dans son domaine. Pour saisir cette différence, observons un énoncé ouvert aux deux interprétations : Tu lui verses l'espoir (Baudelaire). Si l'interprétation est métaphorique, le verbe verser est transféré du domaine des substances concrètes liquides dans le domaine des sentiments humains, où il reçoit comme objet pertinent l'espoir. Le transfert, à son tour, déclenche une interaction entre les deux concept étrangers : du fait qu'il est versé, l'espoir est vu comme s'il était une substance concrète et liquide. Si la métonymie est choisie, par contre, l'objet du verbe verser n'est pas l'espoir, mais le vin qui donne l'espoir. Grâce à la relation cohérente entre le référent textuel pertinent visé — le vin — et l'espoir, le conflit est démantelé, et chaque concept reste ancré à son domaine d'appartenance. Dans la métonymie, donc, il n'y a pas de transfert ${ }^{(2)}$.

A partir du moment où le transfert et la connexion cohérente sont distingués, la suite de l'argumentation s'impose avec la force de la logique.

En premier lieu, la mise en œuvre d'une connexion requiert la présence de deux concepts saturés. Dans notre exemple, un procès - l'espoir ressenti par une personne - est relié à un référent : le vin. Le transfert, par contre, n'est pas soumis à la contrainte de saturation : un concept non saturé peut être transféré aussi facilement qu'un concept saturé : c'est le cas du verbe verser dans notre exemple, qui reçoit son objet direct — l'espoir — dans le domaine d'arrivée.

Ensuite, à partir de cette prémisse il est possible de prévoir un certain nombre de propriétés qualifiantes et discriminantes qui permettent de tracer une frontière nette entre la métonymie et la métaphore (Prandi 2013). La restriction sur la saturation justifie une distribution limitée de la métonymie dans la structure de la

(2) J'utilise ici le terme métonymie dans le sens large de «nébuleuse métonymique », incluant la synecdoque (Bonhomme 2006). Au moment d'établir la distinction avec la métaphore, ce qui est pertinent n'est pas la nature de la relation activée, qui distingue la métonymie au sens strict de la synecdoque, mais l'alternative entre connexion métaphorique et transfert. 
phrase qui s'oppose à la liberté de la métaphore. De la différence entre transfert et connexion découle la différence pertinente pour la suite de notre analyse : dans la métonymie, la pression du conflit investit le foyer conflictuel; dans la métaphore, par contre, elle investit la teneur cohérente.

\subsection{Extensions lexicales et figures}

La métaphore et la métonymie peuvent se réaliser tant comme figures vives que comme stratégies d'extension du signifié d'unités lexicales — mots et expressions figées.

Dans les cas les plus typiques, une figure est l'issue de l'interprétation du signifié conflictuel d'une expression complexe, et notamment d'une phrase. La phrase de Baudelaire Tu lui verses l'espoir, par exemple, présente un signifié conflictuel : le verbe verser reçoit comme objet direct non pas une substance concrète liquide mais un sentiment humain. La figure n'est ni dans le signifié simple d'un mot — du verbe verser ou du nom espoir — ni dans le signifié complexe de la phrase, mais dans une interprétation contingente du signifié complexe de la phrase dans un texte ou dans un acte de communication. La preuve de la dissociation entre signifié de la phrase et figure est que d'un seul signifié nous pouvons tirer des figures différents. L'énoncé Tu lui verses l'espoir, par exemple, a un signifié univoque : la relation conflictuelle entre le verbe verser et le concept d'espoir. Malgré cela, il est ouvert sur deux interprétations, métonymique l'une et métaphorique l'autre. Dans le premier cas, l'espoir est vu comme un effet produit par le vin, qui est la substance réellement versée. Dans le second, le verbe s'applique à son objet direct, ce qui implique qu'il est transféré dans le domaine des sentiments humains : du fait qu'il est versé, l'espoir se change en substance concrète et liquide.

A la différence de la figure, l'extension lexicale produit un changement dans le signifié d'un mot ou d'une expression figée ${ }^{(3)}$. En présence d'extension lexicale, un mot reçoit une nouvelle acception, qui s'ajoute au signifié primitif, motivée soit par un transfert métaphorique, soit par un glissement métonymique. Le mot aile, par exemple, reçoit la nouvelle acception de « appendice latérale d'un bâtiment » par transfert métaphorique : le modèle de la relation entre l'aile et le corps d'un oiseau est transféré à la structure d'un bâtiment. Le signifié du même mot aile subit une extension métonymique lorsqu'il passe de l'acception déjà métaphorique « position latérale de l'attaque d'une équipe », à l'acception « joueur qui occupe la position d'aile ».

Les extensions de signifié se partagent en deux grandes familles, dont les propriétés qualifiantes sont mises en relief par la métaphore.

Les catachrèses de la tradition (Fontanier 1821(1968:39)) dénotent un concept familier par un mot tiré d'un domaine étranger. L'extension, purement régressive, se base sur l'adaptation du concept étranger à la teneur, et demeure isolée, non productive. Un exemple de catachrèse est l'aile du bâtiment. Une fois appliqué au bâtiment, le concept d'aile perd toutes les caractéristiques incompatibles avec l'identité de celui-ci, ce qui bloque la projection. Du fait qu'il a des ailes, par exemple, un bâtiment n'est pas censé voler.

(3) L'expression idiomatique, qui reçoit un signifié figuré en même temps que sa structure syntaxique se fige (Gross 1996), se comporte de la même façon : par exemple passer le Rubicon. 
D'autres extensions, par contre, ne sont pas isolées, mais appartiennent à des réseaux complexes de projections motivées par des concepts métaphoriques actifs intégrés dans notre pensée spontanée (Lakoff, Johnson 1980(2003); Gibbs 1994). Un exemple d'extension qui appartient à un réseau complexe de projections est l'emploi de verser avec l'argent, qui hérite de ce fait une partie de la distribution cohérente des substances liquides : dans le lexique du français, l'argent forme des flux, on peut le verser, le prélever, le congeler, y nager même ; par contre, on ne s'y baigne pas, et il ne forme pas de gouttes ni de vagues. Si de telles connexions sont activées, le résultat est un conflit.

Par-delà cette différence essentielle, les catachrèses isolées et les extensions motivées par des concepts métaphoriques actifs partagent une propriété essentielle, qui les sépare nettement des figures, qui sont des interprétations figurées d'expressions complexes au signifié conflictuel : en tant que signifiés codés de mots ou d'expressions figées, les extensions métaphoriques et métonymiques sont tautologiquement cohérentes du simple fait qu'elles sont partagées par la communauté des locuteurs (Prandi 2010). Dans l'idée d'aile d'un bâtiment ou de versement d'argent, par exemple, il n'y a aucun conflit.

\section{Y a-t-il une «carrière de la métaphore " de la figure à l'extension lexicale?}

Étant donné que les extensions et les figures partagent les mêmes stratégies cognitives, on pense normalement que tant les acceptions tropiques des lexèmes que les signifiés idiomatiques des expressions figées résultent de la cristallisation de figures autrefois vives. Cette idée, lancée par Nietzsche (1873) comme une arme de guerre contre la métaphysique et reprise dans le même esprit par Derrida (1971), traverse en fait 1'histoire de la rhétorique, de Quintilien aux métaphorologies cognitives. Selon Deignan (2005: 40), par exemple, «it seems likely that all conventional linguistic metaphors must have been innovative at some point in history ». Indépendamment de sa valeur empirique, sur laquelle nous reviendrons, cette idée se fonde sur un présupposé : les extensions lexicales et les interprétations figurées ont la même structure ; dans les deux cas, notamment, la figure exercerait une pression conceptuelle sur le foyer ${ }^{(4)}$.

Dans les pages qui suivent, je vais mettre en discussion ce présupposé et proposer une hypothèse qui va dans le sens opposé. Les extensions lexicales et les figures n'ont pas toujours la même structure, étant donné que dans les métaphores vi-

(4) Ce présupposé se manifeste dans sa version la plus radicale dans la conviction qu'une figure attribue au foyer un signifié nouveau, qui dans l'extension lexicale se changerait de création individuelle en valeur partagée par la communauté. L'idée de trope comme changement de signifié parcourt la rhétorique occidentale de Quintilien — « Tropos est uerbi uel sermonis a propria significatione in aliam cum uirtute mutatio » (Institutio oratoria: VIII, 6) - aux métaphorologies cognitives en passant par Black. Tout en bouleversant la vision traditionnelle de la métaphore comme aventure d'un mot, Black reste ancré à l'idée d'un signifié figuré acquis par le foyer : «the focal word [...] obtains a new meaning, which is not quite its meaning in literal uses, nor quite the meaning which any literal substitute would have. The new context imposes extension of meaning upon the focal word » (Black 1954 (1962) : 39). Dans la tradition cognitive, l'idée est implicite dans l'hypothèse d'une continuité de fond entre extensions lexicales et figures. Le prix à payer pour généraliser idée de trope est l'utilisation du terme signifié dans des acceptions différentes : lorsqu'on parle d'extensions, on se réfère au signifié lexical d'un mot ou d'une expression figée ; lorsqu'on parle de figures vives, on se réfère à tour de rôle à la dénotation d'un syntagme nominal référentiel ou à l'interprétation textuelle contingente de toute une phrase. 
ves la pression conceptuelle du trope ne frappe pas le foyer mais la teneur. A partir de cette prémisse, nous pourrons formuler l'hypothèse que les figures et les extensions lexicales sont deux stratégies indépendantes avec une structure et des fonctions différentes.

\subsection{La pression conceptuelle du trope : foyer et teneur}

Quand une extension lexicale se produit, il n'y a aucun conflit : la connexion nourrir un espoir, par exemple, est parfaitement cohérente. Toutefois, l'extension lexicale peut être décrite, à l'instar d'une figure vive, comme une transaction entre un cadre et un foyer. La connexion cohérente nourrir l'espoir, par exemple, a la même structure que l'expression conflictuelle verser l'espoir : le nom espoir appartient au cadre cohérent, alors que le verbe nourrir est le terme focal, transféré du domaine des êtres vivants dans le domaine des sentiments.

Cette précision nous permet de constater que dans l'extension lexicale la pression du trope est canalisée sur le foyer. Dans la connexion nourrir l'espoir, par exemple, c'est le verbe nourrir qui acquiert par métaphore une acception nouvelle, qui fait place à une classe d'arguments différents par rapport aux êtres vivants de l'acception primitive. Le même comportement est documenté par l'extension métonymique. Le mot aile, par exemple, acquiert une acception nouvelle qui fait place à une nouvelle classe de référents : les personnes qui occupent la position d'aile. Dans les deux cas d'extension, le présupposé en discussion est donc satisfait, car c'est le terme focal qui subit la pression du cadre cohérent.

Cette restriction sur l'extension lexicale peut être généralisée, et elle a une motivation fonctionnelle évidente. La fonction d'une extension lexicale n'est pas de changer le profil conceptuel de la teneur sous la pression d'un foyer étranger mais bien d'élargir l'emploi d'un lexème de la langue à des concepts et objets nouveaux. Étant donné la connexion nourrir l'espoir, par exemple, il est fonctionnel d'ouvrir le verbe nourrir aux sentiments, alors qu'il serait absurde de changer les sentiments en êtres vivants. Dans la métonymie, à plus forte raison, il est absurde d'imaginer une pression sur le foyer, car il n'y a pas de transfert. Dans la métonymie de l'aile, par exemple, il est fonctionnel d'inclure les personnes qui occupent la position d'aile dans la dénotation du mot aile, alors que la relation ente la position et la personne empêche de projeter la position sur la personne.

Dans le cas de la métonymie, la figure se comporte exactement comme l'extension. Dans l'expression Il épouserait une grosse dot (Zola), par exemple, le foyer étranger - la dot - subit la pression du cadre cohérent : du modèle conceptuel cohérent du mariage. De ce fait, un référent pertinent visé textuellement - la femme - est identifié en dehors de la classe d'objets désignée par le nom dot. Il est intéressant de remarquer à ce point qu'une fois le référent pertinent recouvré la métonymie admet deux stratégies interprétatives différentes : une stratégie substitutive qui remplace le foyer par la teneur - la femme prend la place de la dot — et une stratégie conservative qui incorpore le foyer et la connexion cohérente - la femme qui porte la dot prend la place de la dot. Dans les deux cas, c'est toujours le foyer qui cède à la pression du cadre. Encore une fois, le présupposé selon lequel la pression conceptuelle du trope frappe le foyer est satisfaite. Pour voir le présupposé remis en question, il faut envisager la métaphore.

Dans le cas des métaphores, le point pertinent pour notre discussion est l'alternative entre une option substitutive et la projection, ce qui nous oblige à réévaluer la question de la substitution. 
En tant que critère définitoire de la figure, la substitution est certainement inadéquate (Black 1954). En tant qu'option textuelle, elle est une option parmi d'autres, mais à une condition non banale, à savoir, à la condition qu'elle soit admise par la structure de la métaphore. En effet, la disponibilité d'un substitut virtuel n'est pas une propriété de la métaphore en tant que telle, mais une propriété de l'expression focale. Pour ne considérer que deux exemples clairs, un nom référentiel métaphorique est par définition substitutif en tant que nom référentiel, car la fonction d'identifier un référent contingent n'est pas liée au signifié de l'expression : à la seule condition que le référent soit identifié, par exemple, l'expression les crapauds du Marais peut être remplacée, entre autres, par l'expression les nobles de Paris. Un nom prédicatif, par contre, n'admet pas d'être remplacé, car sa fonction prédicative est liée à son signifié par une relation biunivoque : dans la phrase Les nobles sont des crapauds, par exemple, le remplacement du prédicat changerait la prédication.

$\mathrm{Si}$, ces précisions faites, nous revenons maintenant à la question de la pression conceptuelle, nous pouvons constater que la substitution, quand elle est admise, est la seule options qui comporte une pression sur le foyer en présence d'une métaphore. Si par contre l'option projective est activée, la pression conceptuelle se déplace sur la teneur. L'expression les crapauds du Marais est une métaphore substitutive quand elle se réfère aux nobles de Paris. De ce fait, elle offre à l'interprète deux options : ou remplacer le foyer par le référent pertinent, ou projeter sur le référent pertinent le concept focal : le crapaud. Dans le premier cas, la pression du cadre cohérent investit le foyer jusqu'à l'expulser. Dans le second, le profil de la teneur est restructuré sous la pression du foyer : les nobles sont vus comme des crapauds.

Pour les métaphores n'admettant aucune option substitutive, la projection, et donc la pression sur le foyer, est la seule issue admise. L'exemple le plus simple est le nom prédicatif : face à une expression comme L'homme est un roseau (Pascal), la seule option est de projeter sur l'homme le système de concepts axé sur le roseau. Le cas le plus intéressant, cependant, est celui du verbe. En tant que terme insaturé, le verbe met en place, nous l'avons vu, deux métaphores logiquement distinctes: l'une axée sur le foyer verbal, auquel s'oppose comme teneur un double virtuel cohérent, et l'autre axée sur l'argument conflictuel, une deuxième teneur à laquelle s'oppose comme foyer l'argument cohérent du verbe. Une phrase au signifié conflictuel comme La lune rêve, par exemple, s'ouvre sur deux métaphores : ou bien un état cohérent non mieux spécifié de la lune est vu comme une instance de rêve, ou bien l'être humain est projeté sur la lune, qui est humanisée. La position des deux métaphores à l'égard de la substitution est très différente. La métaphore axée sur le foyer est virtuellement ouverte à la substitution, qui cependant est moins immédiate qu'en présence d'un nom référentiel et, surtout, est bien plus arbitraire et réductrice : arbitraire, du fait qu'un substitut virtuel du foyer n'est pas toujours immédiatement accessible ; réductrice parce qu'elle bloque l'option complémentaire, à savoir la métaphore axée sur l'argument cohérent. Cela dit, nous pouvons toujours envisager les deux options ouvertes au nom référentiel : la pure et simple substitution ou la projection. Au contraire, la métaphore axée sur la 1'argument cohérent - sur la lune - est incompatible avec toute option substitutive. La teneur soumise à pression introduit dans l'expression un sujet de discours pertinent et cohérent; de ce fait, la pression ne peut pas aboutir à une substitution. La seule option qui demeure est la projection, et donc le déplacement de la pression conceptuelle sur le foyer : l'être humain est projeté sur la lune. 
Il est évident, d'après notre analyse, qu'en présence d'une métaphore vive la pression sur le foyer, limité à la substitution, est une option partielle, car elle n'intéresse qu'une partie des métaphores, et marginale, car elle annule le potentiel projectif et créateur. Si cela est vrai, c'est la projection qui qualifie la métaphore comme figure, et donc la pression sur la teneur. Conférant à la métaphore un statut excentrique et rehaussant son potentiel créateur spécifique et exclusif, le critère de la pression conceptuelle bouleverse la topographie du champ tropologique. Au jour de ce critère, en particulier, il y a moins de différence entre une extension métaphorique et une extension métonymique d'une part, et entre une métonymie et une métaphore substitutive d'autre part, qu'entre une extension métaphorique et une métaphore vive et projective.

\subsection{L'espace de la création}

Du fait qu'elles sollicitent le foyer, les structures ouvertes à la substitution sont les mêmes qui s'ouvrent à l'extension : dans le premier cas, le foyer est remplacé par la teneur; dans le second, il lui fait place. Opposant extension et substitution et par là catachrèse et figure, la tradition classique n'envisage ni la projection ni la pression sur la teneur. Tout en faisant place à la projection, les métaphorologies cognitives ne vont pas au-delà non plus. Selon Lakoff, Turner (1989 : 26), les figures s'enracinent dans le même sol de concepts métaphoriques partagés qui alimente les extensions lexicales, ce qui fait que « a continuum exists between creative and conventional use of metaphor » (Freeman 2007 : 1185). La conséquence directe est que la pression sur la teneur n'est pas envisagée. Or, tant que la pression conceptuelle investit le foyer, et donc l'élément étranger qui menace la cohérence, la structure acquise des concepts n'est pas affectée mais simplement assumée telle qu'elle est.

Parmi les structures conceptuelles qui forment la pensée acquise, en effet, on compte aussi bien les réseaux de relations conceptuelles qui sous-tendent les glissements métonymiques que les concepts métaphoriques partagés qui motivent les extensions lexicales. Au sujets des métonymies, il faut souligner que tant les extensions que les figures vives se basent sur les mêmes réseaux de relations indépendantes, qui feraient partie de notre paysage cognitif même si aucune métonymie n'était mise en place. La relation qui permet d'interpréter la métonymie vive très forte de Zola Il épouserait une grosse dot — la relation entre l'épouse et la dot — n'a pas une nature différente de la relation entre le contenant et le contenu qui motive l'emploi Boire un verre. Il en est tout autrement pour la métaphore. Une extension lexicale comme l'emploi de cultiver avec le nom idée en position d'objet direct repose sur une métaphore cohérente et partagée qui fait partie d'un équipement conceptuel accessible préalablement. L'interprétation des métaphores vives, au contraire, se base sur un mécanisme spécifique de transfert et projection inséparable de la métaphore et mis en place par elle, qui affecte en profondeur la conceptualisation.

Les formes de création actives dans l'interprétation des métaphores vives engagées dans le jeu de la poésie n'ont pas de conséquences durables sur la structure de nos concepts de long terme : une expression comme Le soleil versait à grands flots sa lumière sur le Mont Blanc (H. B. de Saussure), par exemple, nous autorise à voir la lumière comme une substance liquide et à projeter sur la lumière tout le réseau de concepts cohérent avec une substance liquide (\$2.2); en même temps, notre conception de long terme de la lumière n'est pas affectée. Mais nous savons que la création métaphorique ne se réduit pas à la poésie : plusieurs concepts philosophiques et scientifiques et plusieurs termes de spécialité naissent en effet de l'inter- 
prétation métaphorique cohérente d'expressions conflictuelles créées par un auteur. Dans ces cas, l'issue d'une création métaphorique est acquise à la pensée de long terme. Le concept de sélection naturelle (Darwin 1859(1950) est un bon exemple. Au départ, il s'agit d'une connexion conflictuelle : la sélection attribuée à la nature est une action humaine accomplie par un agent à la poursuite d'un but intentionnel ; le modèle projeté sur la nature est le «fermier clairvoyant » qui sélectionne son bétail et contrôle sa reproduction pour améliorer sa qualité. Certes, à la fin du parcours le terme sélection acquiert une nouvelle acception relevant du domaine de la biologie. Ce changement de signifié, cependant, n'est pas l'issue d'une pression du cadre cohérent sur le foyer, comme il arrive dans l'extension lexicale, mais le résultat d'un processus de projection et donc de création (Prandi, Rossi 2012). Dans le parcours interprétatif qui mène du conflit au concept cohérent, la pression conceptuelle est d'abord canalisé sur la teneur : il n'est pas question d'adapter le concept de sélection à la nature organique, mais bien de remettre en question une conceptualisation acquise de la nature. La cohérence du concept scientifique n'est ni une donnée tautologique, comme la cohérence des extensions lexicales - par exemple, cultiver un projet - ni l'issue d'une adaptation régressive du foyer à la teneur, mais l'issue d'une restructuration du concept de nature d'après le modèle de la sélection ${ }^{(5)}$. Les concepts de la science et de la philosophie montrent que la créativité métaphorique ne se déploie pas seulement dans le jeu poétique, où elle n'a pas de conséquences durables sur la structure des concepts, mais aussi dans le domaine de la pensée cohérente et durable. Grâce à la capacité de l'expression de connecter les concepts dans des relations inattendues, voire conflictuelles, la pensée cohérente peut sortir de ses routines et s'ouvrir à la création.

\subsection{Deux sources pour la métaphore}

Le critère de la pression conceptuelle fait tomber le présupposé qui sous-tend l'idée d'une origine commune des extensions lexicales et des figures vives : ce n'est pas vrai que tous les tropes, métaphores et métonymies, extensions lexicales et figures vives, canalisent la pression conceptuelle du trope sur le foyer. Ce point n'a pas de retombées sur les métonymies, mais il entraîne des conséquences profondes pour l'étude des métaphores. En particulier, il encourage à remettre en question l'idée d'une « carrière de la métaphore » de la figure à l'extension lexicale.

Notre ligne argumentative n'exclut pas qu'une métaphore vive puisse se cristalliser et se changer en signifié codé. Simplement, on ne peut pas assumer sans autre une telle conversion comme allant de soi sur le fond d'une présupposition de

(5) L'idée que la formation de concepts cohérents à partir d'un conflit finit par affecter à tour de rôle tant le foyer que la teneur pourrait faire penser à un cas de blending, ou conceptual integration (Fauconnier, Turner 1998; 2002; Turner 2007). Mais il y deux caractéristique de la projection telle qu'elle est décrite dans cet essai qui nous éloignent de l'idée de blending. En premier lieu, la création n'est pas un processus interne au périmètre de la pensée cohérente — de la cognition — mais passe par un conflit conceptuel rendu possible par le pouvoir de mise en forme d'une expression linguistique. Ensuite, le concept cohérent issu d'un acte de création métaphorique n'est pas simplement un «blended space » qui contient éléments de deux «input spaces » : l'idée de sélection naturelle, par exemple n'est pas un mélange qui hérite quelques propriétés du concept reçu de nature et quelques propriétés du concept de sélection, mais l'issue d'un processus qui se déroule en deux phases dont la structure peut être décrite avec exactitude : une première phase, la projection, sollicite la teneur avec des inférences étrangères mais fécondes; la seconde adapte le concept focal de sélection non pas à l'idée de nature qui précède la métaphore mais à une idée préalablement refaçonnée par la projection. 
continuité, mais il faut l'envisager comme une question empirique et, le cas échéant, identifier des exemples parmi les faits de langue. Qu'il s'agisse d'une question empirique, et non pas d'une impossibilité logique, est prouvé par la présence de conversions comparables, qui sont attestées dans au moins deux cas et dans les deux sens.

Le passage des concepts scientifiques et philosophiques de la phase de conflit et de pression sur la teneur à la phase de stabilisation cohérente et donc de pression sur le foyer est largement documenté, et n'a pas échappé à l'attention des épistémologues et des historiens des sciences (voir par exemple Kuhn 1962 ; 1979(1993); Hesse 1965(1966) ; Boyd 1979). Hesse (1965(1966 : 160)), par exemple, souligne que les métaphores qui fournissent une « voie d'accès épistémique » (Boyd 1979 : 377) à des domaines scientifiques passent forcément par une phase de conflit destinée à remettre en question les idées reçues : «it is necessary that there should be patent falsehood or even absurdity in taking the conjunction literally. Man is not, literally, a wolf; gases are not in the usual sense collections of massive particles ». Le conflit autorise à projeter sur la teneur une constellation d'inférences qui ne serait pas accessible en dehors de la métaphore. C'est la phase où le système de concepts axé sur le foyer sollicite la teneur : le concept de sélection, par exemple, force l'idée consolidée de nature. En même temps, les inférence projetables sont soumises à la discussion de la part de la communauté des savants et retenues en fonction de leur accessibilité au contrôle empirique sur le fond d'un critère de cohérence. C'est la phase où la pression se déplace sur le sujet subsidiaire, qui est redéfini pour faire place à de nouveaux contenus : le terme sélection, par exemple, acquiert une nouvelle acception dans le domaine spécialisé de la biologie. Ces passages peuvent être documentés au cas par cas dans 1'histoire des différentes disciplines.

Une conversion de sens opposé a lieu chaque fois qu'une métaphore conventionnelle est revitalisée. L'idée que la vérité est une lumière, par exemple, est aussi vieille que la culture occidentale. Normalement, sa réception est automatique et ne comporte aucun processus créateur. Une reprise de cette métaphore par Alexis de Tocqueville, cependant, finit par projeter sur la vérité une propriété de la lumière qui sort des clichés culturels, à savoir la fragilité : La vérité est pour moi [...] une lumière que je crains d'éteindre en l'agitant. Une fois associée à la vérité, la fragilité déclenche à son tour des inférence de responsabilité et de soin, dans une chaîne qui est typique de la projection.

Les cas analysés montrent que si l'on veut défendre l'idée d'une « carrière de la métaphore » de la figure vive à l'extension partagée il faut creuser l'histoire des langues et vérifier si et dans quelle mesure de telles conversions sont attestées. Si des cas sont documentés, il faut décrire exactement les phases différentes qui se sont succédées, et identifier le moment où l'orientation de la pression conceptuelle s'est renversée. Cela dit, il apparaît franchement peu plausible que la seule source, voire la source principale des extensions lexicales cohérentes soit à chercher dans la cristallisation de métaphores créatives. Et cela pour plusieurs raisons.

Une analogie avec la création des concepts spécialisés des sciences est déroutante. Les concepts spécialisés naissent d'actes de création individuelle finalisés, ce qui fait que tant le conflit séminal que l'évolution vers la cohérence sont logiquement requis et en principe accessibles à la description empirique. Les extensions lexicales, par contre, sont l'issue d'un processus anonyme pour lequel il est franchement difficile d'imaginer et de documenter tant un conflit séminal, qui serait par définition un acte individuel, qu'une évolution vers la cohérence. 
Les concepts métaphoriques cohérents sont partagés par une communauté culturelle qui s'étend dans l'espace et dans le temps bien au-delà des frontières d'une communauté linguistique donné. Cette propriété, qui est la véritable raison d'être du tournant cognitif en métaphorologie, ne se justifie pas dans l'hypothèse d'une création individuelle dans les limites d'une langue donnée, alors qu'elle est tout à fait cohérente avec l'hypothèse que les concepts métaphoriques sont une composante essentielle de l'équipement de base des êtres humains documenté dans plusieurs langues et mêmes dans des traditions culturelle différentes et dans des moments différents de l'histoire. De plus, l'idée que le patrimoine d'acceptions figurés qui enrichit le lexique de nos langues se réduit à une espèce de cimetière plein d'os desséchés d'anciennes figures désormais usées est en contradiction manifeste avec la vitalité intacte du riche patrimoine de concepts métaphoriques qui organisent d'entiers domaines de notre expérience et de notre pensée quotidienne.

Finalement, si nous jetons un coup d'œil dans les profondeurs de l'histoire, nous nous rendons compte que les motivations métaphoriques des extensions lexicales qui nous sont familières sont attestées depuis longtemps, alors même que les métaphores vives documentées depuis l'antiquité sont bien loin de perdre leur éclat au fil des siècles.

Le lexique des sentiments en latin dévoile un paysage métaphorique tout à fait familier (Fedriani 2011). Pour les latins, la peur gèle : Mihi frigidus horror / membra quatit (Virgile) ; Ulixi cor frixit prae pavore (Livius Andronicus). Le désir mord - Sin autem tandem libertatis desiderium remordet animos (Livius) - et incendie le sujet - Erant quos memoria Neronis ac desiderium prioris licentiae accenderet (Tacite), qui brûle ou explose : Ille vir [...] flagrat, ardet cupiditate iusti et magni triumphi (Cicéron). On peut garder un désir dans l'esprit comme une chose précieuse - Repositum in animo nostro desiderium (Seneca) - mais on peut aussi tomber dedans : Cum in imperiorum honorum gloriae cupiditatem inciderunt (Cicéron). L'espoir est localisé dans le sujet — Spes est in vobis (Cicéron : Hope is in you) — qui le nourrit : Spem alicujus alere (Cicéron). Malgré cela, il s'empare du sujet - Magna me spes victoriae tenet (Sallustius) et le secoue : Cuius animus spe, metu, admonitione, precibus, vanitate denique [...] agitandus est (Quintilien). L'objet de l'espoir est un lieu où il est placé - Spem ponere in armis (Virgile) — où duquel il peut être enlevé : Spem deponere (Horace). Et ainsi de suite.

Si nous nous penchons sur les métaphores vives, par contre, nous constatons que les millénaires ne suffisent pas à faner leur éclat. Dans la littérature latine nous trouvons quelques traces d'une métaphorique de la lumière liquide destinée à connaître ses moments de splendeur pas moins de deux millénaires plus tard. Aennius parle de Liquidas [...] aetheris ora, alors que Lucrèce définit le soleil comme une source de lumière liquide : Liquidi fons luminis [...] sol. Dans le romantisme anglais et dans le symbolisme français, la lumière liquide est encore une métaphore fraîche qui connaît toutes ses métamorphoses imaginables (Prandi 2012) : elle se verse et coule en torrents et rivières, forme des gouttes et de vagues, baigne et submerge, et ainsi de suite :

They pour fresh light (Shelley); The moon rains out her beams (Shelley); The light that flow'd down on the winds (Blake); The light which streams here (Byron); The free heaven [...] rains fresh light and dew / On the wide earth (Shelley); Liquid streams of light (Shelley); The floods of light / Which flow over the world (Shelley); The sunset's sea of beams, / Whose golden waves [...] / Fade fast (Shelley); Lo! The light of the morning is flowing / Through radiant portals of gold (Char- 
lotte Brontë). Le ciel bas et lourd [...] / il nous verse un jour noir plus triste que les nuits (Baudelaire); L'astre coule et fait un ruisseau (Leconte Delisle); Il est étendu dans l'herbe, ...] / pâle dans son lit vert où la lumière pleut (Rimbaud) ; La pleine lune s'étalait, / et la solennité de la nuit, comme un fleuve, sur Paris dormant ruisselait (Baudelaire); La lune est dans son plein, / d'une blanche lueur la clairière est baignée (V. Hugo); Torrent / de lumières prodigués dans un commun et rapide écoulement (Michelet); Comme un flot ruisselant [...] de lumière (De Banville); L'astre coule et fait un ruisseau (Leconte Delisle).

Cela montre que les métaphores vives, et avec elles les conflits conceptuels qui les rendent possibles, peuvent franchir plusieurs barrières temporelles, linguistiques et culturelles sans se faner. Et on n'arrive même pas à imaginer le moment où la lumière liquide deviendrait un concept métaphorique cohérent et partagé, une «métaphore de la vie quotidienne ».

S'il est vrai qu'on ne peut pas exclure une conversion, il y a davantage d'arguments pour conclure que deux ordres de structures indépendantes coexistent dans le terrain des métaphores : d'une part, un patrimoine anonyme de concepts métaphoriques cohérents et partagés qui alimentent les extensions lexicales; d'autre part, la construction d' expressions signifiantes au contenu conflictuel prêtes à alimenter tant les métaphores vives de la poésie que la création métaphorique de concepts cohérents en science, en philosophie et en terminologie. Les métaphores vives naissent d'actes de création individuelle, alors que les concepts métaphoriques témoignent d'une habilité cognitive enracinée dans la pensée spontanée et appartiennent à un patrimoine anonyme et partagé qui se manifeste dans les structures lexicales des langues.

\section{Conclusion}

Dans la vision héritée par une tradition plus que bimillénaire, l'unité du champ tropologique se fonde sur deux présupposés : tout trope réalise un transfert de concepts, qui se résout au moment où le terme focal est adapté à la cohérence de la teneur. Sur ces présupposés, la métaphore et la métonymie, la figure et l'extension lexicale partagent les mêmes propriétés de fond et sont donc directement comparables. Un corollaire traditionnel de cette vision est que les extensions lexicales motivées par des stratégies métaphoriques ou métonymiques sont l'issue de processus de cristallisation de figures autrefois vives.

Si nous remettons en question les deux présupposés, l'unité du champ tropologique est brisée.

D'une part, seule la métaphore réalise un transfert de concepts, alors que la métonymie exploite une connexion cohérente entre des concepts qui demeurent chacun ancré à son domaine d'appartenance.

D'autre part, s'il est vrai que les métonymies et les extensions lexicales métaphoriques canalisent la pression conceptuelle sur le foyer, qui se voit remplacé ou redessiné, les métaphores vives investissent la teneur, dont l'identité conceptuelle est sollicitée.

Sur le fond de cette mise au point, l'idée que les extensions lexicales naissent de métaphores autrefois vives doit être réexaminée. L'hypothèse d'une conversion ne peut pas être exclue a priori, d'autant plus que des cas comparables de glissements de la pression conceptuelle sont attestés. Il s'agit de vérifier sur le plan empirique si et dans quelle mesure l'histoire des langues fournit des exemples. Ce- 
pendant, plusieurs arguments poussent à emboucher un chemin différent, et à penser que dans la métaphore deux ordres de structures indépendantes coexistent : un patrimoine anonyme de concepts métaphoriques cohérents et partagés et la construction d'expressions au contenu conflictuel ouvertes tant à l'interprétation figurée dans un texte qu'à la création de concepts spécialisés de la philosophie, des sciences ou des différentes terminologies.

\section{Bibliographie}

Barcelona, A. (éd., 2000 (2003)), Metaphor and Metonymy at the Crossroads, $2^{\mathrm{e}}$ éd., Mouton de Gruyter, Berlin - New York.

Benczes, R., BARCelona, A., \& Ruiz de Mendoza IbÁÑEZ, F. J. (éds. 2011), Defining Metonymy in Cognitive Linguistics : Towards a Consensus View, John Benjamins, Amsterdam - Philadelphie.

BLACK, M. (1954 (1962)), " Metaphor », Proceedings of the Aristotelian Society 55, 273-294. Réimpr. in M. Black, Models and Metaphors, Cornell University Press, Ithaca - Londres : 25-47.

Bonhomme, M. (1987), Linguistique de la métonymie, Peter Lang, Berne. - (2006), Le discours méthonymique, Peter Lang, Berne.

Bowdle, B. F., Gentner, D. (2005), « The career of metaphor », Psychological Review 112, $1: 193-216$.

BOYD, R. (1979 (1993)), « Metaphor and theory change : what is "Metaphor" a metaphor for? », in Ortony (éd. 1979(1983)) : 481-532.

DARWIN, Ch. (1859 (1950)), On the Origin of Species by means of Natural Selection, A Reprint of the First Edition, Watts \& Co, Londres.

DeIGnan, A. (2005), Metaphor and Corpus Linguistics, John Benjamins, Amsterdam - Philadelphie.

Derrida, J. (1971 (1982)), « La mythologie blanche », Poétique 2. Réimpr. in J. Derrida, Marges de la philosophie, Les Éditions de Minuit, Paris : 247-324.

DirVen, R., Pörings, R. (2002), Metaphor and metonymy in comparison and contrast, Mouton de Gruyter, Berlin - New York.

FAuconnier, G., TURner, M. (1998), « Conceptual integration networks », Cognitive Science, 22, 2 : 133-187.

- (2002), The Way we Think: Conceptual Blending and the Mind's Hidden Complexities, Basic Books, New York.

FEDRIANI, C. (2011), «Experiential metaphors in Latin : Feeelings were containers, movements and things possessed », Transactions of the Philological Society $109,3: 307-326$.

FONTANIER (1821 (1968)), Les figures du discours, Flammarion, Paris. Contient : Manuel classique pour l'étude des tropes (1821.4éd., 1830) et Traité général des figures de discours autres que les tropes (1827).

GiBBS, R. W. (1994), The Poetics of Mind, Cambridge University Press, Cambridge. 
Gross, G. (1996), Les expressions figées en français, Ophrys, Gap - Paris.

Henry, A. (1971), Métonymie et métaphore, Klincksieck, Paris.

Hesse, M. B. (1965 (1966)), « The Explanatory Function of Metaphor », in Y. BarHillel (éd.), Logic, Methodology, and Philosophy of Science, North Holland, Amsterdam. Réimpr. in M. B. Hesse, Models and analogies in science, University of Notre Dame Press, South Bend : 157-177.

KuHn, Th. S. (1962), The Structure of Scientific Revolutions, The University of Chicago Press, Chicago - Londres.

— (1979 (1993)), « Metaphor in Science », in Ortony (éd., 1979(1993)), 533542.

LAKOFF, G. (1987), Women, Fire and Dangerous Things, The University of Chicago Press, Chicago - Londres.

LAKOfF, G., Johnson, M. (1980), Metaphors we Live by, The University of Chicago Press, Chicago - Londres.

LAKoff, G., TURner, M. (1989), More than Cool Reason, The University of Chicago Press, Chicago - Londres.

LE GuERn, M. (1973), Sémantique de la métaphore et de la métonymie, Larousse, Paris.

LOUNSBURY, F. L. (1964), "A semantic analysis of the Pawnee kinship usage », Language $32: 158-194$.

NiETzSCHE, F. (1873(1999)), « Ueber Wahrheit und Lüge im aussermoralischen Sinne », in Die Geburt der Tragödie. Unzeitgemäße Betrachtungen I-IV. Nachgelassene Schriften 1870-1873. Edition critique : Sämtliche Werke. Kritische Studienausgabe in 15 Einzelbänden par G. Colli et M. Montinari, Band 1, De Gruyter, Berlin - New York : 871-890. Ortony

PRANDI, M. (1992), Grammaire philosophique des tropes, Les Editions de Minuit, Paris.

— (2004), The Building Blocks of Meaning, John Benjamins, Amsterdam - Philadelphie.

— (2010), «Les expressions idiomatiques en italien : entre arbitraire et motivation », in M. H. Araújo Carreira (a cura di), L'idiomaticité dans les langues romanes, Université Paris 8 - Vincennes Saint Denis, Travaux et documents 48 : 395-406.

- (2012), «A Plea for Living Metaphors : Conflictual Metaphors and Metaphorical Swarms », Metaphor and Symbol 27, 2 : 148-170.

— (2013), « La différence entre métaphore et métonymie sous le jour du conflit conceptuel », L'information grammaticale $137: 14-20$.

PRANDI, M., Rossi, M. (2012), « Les métaphores dans la création de terminologie : quelques perspectives ouvertes », Terminologie : textes, discours et accès aux savoirs spécialisés, Glat-Genova 2012, Télécom Bretagne, Brest : 7-18.

QUINTILIEN, Institutio oratoria - De l'institution oratoire, VIII, Paris, les Belles Lettres, 1978.

RICHARDS, I. A. (1936), The Philosophy of Rhetoric, Oxford University Press, Oxford.

TURner, M. (2007), «Conceptual Integration », in D. Geeraerts, H. Cuyckens (Eds.), The Oxford Handbook of Cognitive Linguistics Oxford University Press : 377393. 